NASA Contractor Report 204137

ICOMP-97-06; CMOTT-97-01

AIAA-97-3243

\title{
Application of Low Dimensional Manifolds in NOx Prediction
}

\section{A.T. Norris}

Institute for Computational Mechanics in Propulsion

and the Center for Modeling of Turbulence and Transition

Cleveland, Ohio

August 1997

Prepared for

Lewis Research Center

Under Cooperative Agreement NCC3-534

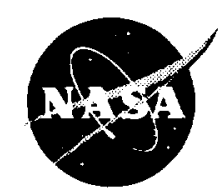

National Aeronautics and

Space Administration

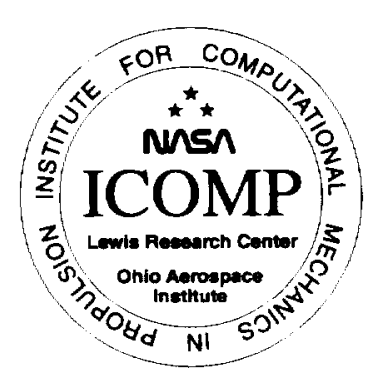


- 


\title{
APPLICATION OF LOW DIMENSIONAL MANIFOLDS IN NOx PREDICTION
}

\author{
A. T. Norris * \\ Institute for Computational Mechanics in Propulsion \\ and the Center for Modeling of Turbulence and Transition \\ NASA Lewis Research Center, Cleveland Ohio.
}

\begin{abstract}
A new post-processing technique has been developed, based on the Intrinsic Low Dimensional Manifold (ILDM) method of Maas and Pope ${ }^{1,2}$.

The ILDM method is a dynamical systems approach to the simplification of large chemical kinetic mechanisms. By identifying low-dimensional attracting manifolds, the method allows complex full mechanisms to be parameterized by just a few variables: In effect, generating reduced chemical mechanisms by an automatic procedure. These resulting mechanisms however, still retain all the species used in the full mechanism.

The NOx post-processor takes an ILDM reduced mechanism and attempts to map this mechanism to the results of a CFD calculation. This mapping allows the NOx concentrations at each grid node to be obtained from the ILDM reduced mechanism, as well as other trace species of interest. Because a mapping procedure is used, this method is very fast, being able to process one million node calculations in just a few minutes.
\end{abstract}

\section{Introduction}

The ability to predict NOx in CFD calculations of reacting flows is constrained by the trade-off between accuracy and available computing resources.

\footnotetext{
* Senior Research Associate, Member AIAA

Copyright (c) 1997 by the American Institute of Aeronautics and Astronautics, Inc. No copyright is asserted in the United States under Title 17, U.S. Code. The U.S. Government has a royalty-free licence to exercise all rights under the copyright claimed herein for Governmental Purposes. All other rights are reserved by the copyright owner.
}

For fluid design calculations, it is enough to get the values of density and temperature correct, and so fairly simple chemical mechanisms may be employed, with good results.

However the prediction of trace species, such as NO, requires the use of a significantly more complex kinetics scheme, with the resulting increase in computational effort required to solve the problem. For example, a simple two-step mechanisn will have six species (Fuel, $\mathrm{H} 2 \mathrm{O}, \mathrm{CO}, \mathrm{CO} 2, \mathrm{O} 2, \mathrm{~N} 2$ ) and two rate equations. To predict NO by the Zeldovich mechanism will require adding 5 more species $(\mathrm{OH} \mathrm{O} \mathrm{H} \mathrm{N}$ $\mathrm{NO}$ ) and six more rate equations. Clearly this has more than doubled the complexity of the problem and the CPU time required.

To overcome this increase in complexity, postprocessing is an attractive option, with the promise of quickly obtaining the NOx fields when the solution of the major species and velocity fields has converged. To do this, two main approaches are employed.

In the first, the composition and temperature at each node are used to obtain the equilibrium value of NOx. This has the advantage of being very quick, but will naturally over-predict the value of NOx at each point. Due to the very slow rate of NOx formation under most conditions, it may significantly over-predict the value. The advantage of this method is its speed, and that it does not need to know anything about the composition at neigbouring nodes.

The second technique employed is an iterative, frozen-composition method. In this the values of velocity, density and major species compositions are frozen and the species transport equations are 
solved for the minor species. The disadvantage of this technique is the time required to perform the iterations to convergence. Typically this can be of a similar CPU time as the original calculation. In addition, the technique is not code independent. i.e. The CFD results from an unstructured code will have to be processed differently than those from a structured code. The advantage is that the NOx reaction rates are not assumed to be fast, and so finite rate effects are accounted for.

In this paper, an approach is outlined that attempts to provide the equilibrium approach with the finite rate effects available to the iterative scheme.

To achieve this, an Intrinsic Low Dimensional Manifold (ILDM) simplification of a full mechanism is performed, so that all species are represented as functions of a mixture fraction $\xi_{I}$ and a progress variable $Y p_{I}$. Then for each node in the CFD data, a mixture fraction $\xi_{C}$ and progress variable $Y p_{C}$ is obtained and by mapping the ILDM variable onto the CFD values, a value for the trace species may be obtained.

\section{ILDM Method}

The ILDM method employed in this work is that used in the NASA Lewis ILDM code. ${ }^{3}$ In this code, the ILDM simplified mechanism is obtained by a trajectory-generated technique, described by Pope and Maas ${ }^{4}$. In this method, the full mechanism is parameterized by two scalars, a mixture fraction based on atom concentrations and a progress variable, based on either species mass fractions, temperature or Gibbs function. The resulting species concentrations, rates and properties are stored in look-up tables. A more detailed description can be obtained by Norris ${ }^{3}$.

One concern in the creation of the ILDM tables is that because the reaction rate of NOx is very slow, the ability to interpolate accurately may be compromised near the fully burnt limit of $Y p_{I}$. This is because the progress variable will only change a small amount, while the NOx values can change by an order of magnitude. To counter this, the look-up table has been modified to cluster the nodes near the fully burnt value. In addition, the choice of temperature as the progress variable is found to give the best resolution for NOx values.

\section{Post-Processor}

The post-processor performs the functions of reading in the CFD data, obtaining the values of $\xi_{C}$ and $Y p_{C}$, and then mapping the composition at each node to the manifold.

The mixture fraction of the CFD data can be obtained by several methods, each based on atom population. The mixture fraction based on the $m$ atom, $\xi_{m}$ is given by

$$
\xi_{m}=\frac{Y_{m}-Y_{m, a i r}}{Y_{m, f u e l}-Y_{m, a i r}}
$$

where $Y_{m}$ is the mass fraction of the $m$ atom, $Y_{m, f u e l}$ is the mass fraction of the $m$ atom in the fuel stream and $Y_{m, f u e l}$ is the mass fraction of the $m$ atom in the air stream.

The three options for progress variable are species, temperature or Gibbs function based. The species option allows one to obtain the progress variable as a normalized mass fraction of a chemical species, or the sum of several chemical species. For example one could chose a mixture fraction based on the mass fraction of $\mathrm{CO} 2$, or the sum of the mass fractions of $\mathrm{CO} 2$ and $\mathrm{H} 2 \mathrm{O}$. The only restriction on this choice is that the resulting quantity should be single-valued. For this reason, a progress variable based on the mass fraction of $\mathrm{OH}$ would not be acceptable, as the $\mathrm{OH}$ value rises, peaks and then drops down to an equilibrium value. Another option is to use temperature as a progress variable, while the Gibbs function provides the third option. In all of these three cases, the progress variable must be the same as the one used to obtain the ILDM table.

Having obtained $\xi_{C}$ and $Y p_{C}$ for each CFD data point, the NOx value is simply obtained by interpolating from the ILDM table at the equivalent $\xi_{I}$ and $Y p_{I}$ locations.

\section{Test Cases}

Three test cases were employed to evaluate the performance of the post-processor: A hydrogenair can combustor, a methane-air combustor and a jeta-air problem.

The hydrogen-air combustor consists of a stepped tube, with swirling air being injected in radially. Hydrogen fuel is injected in downstream of the air inlet, without swirl. Initial conditions for this case 
are air at $804 \mathrm{~K}$, hydrogen at $305 \mathrm{~K}$ and a pressure of $20 \mathrm{~atm}$. In addition, several other inlet conditions were investigated, corrosponding to different operation conditions.

Due to the sensitive nature of the methane and Jet A cases, the configuration of these problems will not be reported. However the initial conditions for the methane case are a fuel inlet temperature of $305 \mathrm{~K}$, an air temperature of $950 \mathrm{~K}$ and a pressure of 20 atm. The Jet A case has initial air temperatures of $740 \mathrm{~K}$, with a fuel temperature of $350 \mathrm{~K}$ and a pressure of $10 \mathrm{~atm}$.

Data available for these flows consists of experimental measurements of NOx levels at the exit plane of the hydrogen-air cases, while the methane and Jet A configurations have the values of NOx obtained at each data point by the inclusion of the Zeldovich mechanism in their reduced mechanism. It should be noted that the CFD results for all but the first hydrogen-air setup were not run to a fully converged condition. However the results are included for completeness.

The full mechanisms used to obtain the ILDM tables are the hydrogen-air mechanism of Miller and Bowman ${ }^{5}$, the methane-air mechanism of Miller and Bowman ${ }^{5}$ and the Jet A-air mechanism of Kollrack ${ }^{6}$.

\section{Results}

To address the accuracy of the mapping procedure, the scalar fields for known major species were extracted by the postprocessor and compared to the CFD results. The mixture fraction was obtained based on the mass fraction of the $\mathrm{H}$ atom for the $\mathrm{H} 2$-air case, and the $\mathrm{C}$ atom for the methane and jet $A$ cases. In all cases, the temperature was used as the progress variable.

For the hydrogen-air Case 1, the predicted mass fraction of $\mathrm{H} 2 \mathrm{O}$ is plotted against the CFD results in Fig 1. As can be seen, there is a good agreement between the CFD result and the manifold prediction. For the methane case, the predicted mass fractions of $\mathrm{CO} 2$ and $\mathrm{H} 2 \mathrm{O}$ are compared to the $\mathrm{CFD}$ results in Fig 2. and 3. respectively. Again there is very good agreement, except for the very low values. It can be seen that the values of $\mathrm{CO}_{2}$ are underpredicted while the mass fractions of $\mathrm{H} 2 \mathrm{O}$ are overpredicted. For the Jet A example, the predicted mass fractions of $\mathrm{CO}_{2}$ and $\mathrm{H} 2 \mathrm{O}$ are compared to the CFD results in Fig 4. and 5. respectively. In this case, the values of $\mathrm{CO} 2$ are underpredicted for the entire range of values, while the $\mathrm{H} 2 \mathrm{O}$ values are overpredicted. This deviation in the results will be discussed in the next section.

For the hydrogen-air cases, the NO exit concentration was predicted and compared to experimental data. The results of this are shown in Table 1. It can be seen that a reasonable agreement is obtained in all 5 cases. It should be noted that the first case is the only one to have converged fully, thus the results for the other four may be somewhat suspect. For the hydrogen case, no CFD values of NOx were available.

$$
\text { Hydrogen-Air NO Prediction Performance. }
$$

\begin{tabular}{|c|c|c|}
\hline Case Number & Prediction & Experiment \\
\hline Case 1 & $20.5 \mathrm{ppm}$ & $21.6-26.5 \mathrm{ppm}$ \\
\hline Case 2 & $6.9 \mathrm{ppm}$ & $10.6 \mathrm{ppm}$ \\
\hline Case 3 & $4.9 \mathrm{ppm}$ & $12.0 \mathrm{ppm}$ \\
\hline Case 4 & $13.9 \mathrm{ppm}$ & $3.1 \mathrm{ppm}$ \\
\hline Case 5 & $28.5 \mathrm{ppm}$ & $35.1 \mathrm{ppm}$ \\
\hline
\end{tabular}

For the methane and JetA case, there are no experimental results available, and so the NOx predicted by the post-processor was compared to the CFD values.

In the Methane case, a reasonable agreement was obtained between the the prediction and the CFD results. A small percentage of results were underpredicted, and can be seen as the band of points lying to the left of the diagonal line in Fig 6 .

For the JetA case shown in Fig. 7 , the predicted NOx does not agree well with the CFD data, except at the high concentration levels.

\section{Discussion}

The results described in the previous section will now be discussed.

For the hydrogen-air case, a very good agreement was obtained between the predicted $\mathrm{H} 2 \mathrm{O}$ values and the CFD results. In addition, the predicted values at the combustor exit plane showed very good agreement with the experimental value. The cost of doing the ILDM prediction was only a few minutes to generate the look-up table and a negligible amount of CPU time to perform the interpolation. For this case then, the procedure looks very promising.

For the Methane case, the $\mathrm{CO} 2$ and $\mathrm{H} 2 \mathrm{O}$ results were very good, except at low concentrations. This 
is caused by a combination of two factors. Firstly, the low concentration values correspond to very lean or unburnt compositions. Under these conditions, it is likely that mixing is a more important process than reaction, and so the ILDM table will not represent the state of the composition well. It is possible to include mixing in the table generation process, which may help this problem, but it has not been done in this case. The second factor is that the reduced mechanism may not agree with the ILDM scheme in certain regions. This would result in the compositions at the same progress variable differing between the two methods, and so the mapping not agreeing.

The comparison of the NO values for the methane case does not exhibit the nice one-to-one agreement of the major species. However it should be considered that the majority of data points do agree within an order of magnitude at the worst, and the high concentration values by a factor of 2 . In addition, the values obtained by the CFD results can by no means be considered a true solution.

For the Jet $A$ case, there is not a very good agreement between the predicted and the CFD results for the major species. In this case, it is probably the full mechanism from which the ILDM table was obtained that is at fault. This mechanism is very old, and contains many outdated values for the individual rate expressions. In addition, the values of NO are only obtained by a Zeldovich mechanism, rather than the more complete mechanisms used in the hydrogen and methane cases. Despite the bad agreement though, the predicted NO values did show the right trend.

Because of the non-itterative nature of this postprocessing technique, the transport, (or advection) of $\mathrm{NOx}$ is not accounted for explicitly. To illustrate this point, consider a $1 \mathrm{D}$ combustion problem. Initially, the fuel and air have mixed and start to react. Moving downstream the composition approaches the fully burnt state, and after sufficent time reaches it. In this example, the NOx postprocessor would show the correct rise in NOx level, until the fully burnt state was reached. After the fully burnt state was reached, the post processor would predict a constant NOx concentration. However in reality, the NOx levels would continue to climb because the time scales of the NOx production are much slower than those of the major reaction components. The conclusion to be drawn from this is that this method will work best for short residence time problems.

For another case, consider the previous example, but at some distance downstream add a dilution jet. For this example, the NOx post-processor would show the correct evolution of the NOx level until the dilution jet. After the dilution jet the post=processor would predict a sudden drop in the NOx concentration, due to the sudden change in composition. However what one would expect to happen would be a gradual change in the NOx levels, due to the slow NOx reaction rates. From this example, it can be concluded that the post processor can be expected to fail when there is sudden changes in the composition of the mixture, except at the initial conditions.

\section{Conclusions}

A very fast and reasonably accurate technique has been developed to post-process CFD combustion results and obtain an estimate of the NOx levels throughout the field. The method offers a significant improvement over iterative methods in CPU time required, while providing a better prediction than equilibrium techniques. However the method is restricted to cases which have relatively short residence times, and that do not exhibit abrupt changes in composition or temperature.

For the three test cases considered in this paper, the hydrogen-air and methane-air examples showed good agreement with available data. The Jet A example did not perform as well, but the deficiencies can be blamed on the poor performance of the full mechanism used to create the look-up tables.

Further work will be required to validate this method, as well as the development of better full mechanisms for Jet A-air reaction. 


\section{References}

1. U. A. Maas and S. B. Pope. Simplifying chemical kinetics: Intrinsic low-dimensional manifolds in composition space. Combustion and Flame, 88 $(3 / 4): 239-264,1992$.

2. U. Maas and S. B. Pope. Laminar flame calculations using simplified chemical kinetics based on intrinsic low-dimensional manifolds. In TwentyFifth Symposium (International) on Combustion, page In Press, 1994.

3. A. T. Norris. Automated simplification of full chemical mechanisms

- In 33rd AIAA/ASME/SAE/ASEE Joint Propulsion Conference, Seattle, Washington, 1997. AIAA-97-3115.

4. S. B. Pope and U. Maas. Simplifying chemical kinetics: Trajectory-generated low-dimensional manifolds. Technical Report FDA-93-11, Sibley School of Mechanical and Aerospace Engineering: Fluid dynamics and aerodynamics program, 1993.

5. J. A. Miller and C. T. Bowman. Mechanism and modeling of nitrogen chemistry in combustion. Prog. Energy Combust. Sci, 15 :28i-338, 1989.

6. R. Kollrack. Model calculations of the combustion product distributions in the primary zone of a gas turbine combustor. In ASME Winter Annual Meeting, New York $N Y$, pages ASME 76-WA/GT-7, 1976.

\section{Acknowlegements}

The author would like to thank Dr. A. Brankovic of Pratt and Whitney for providing the test examples for this paper, and for obtaining the JetA-air mechanism. Also thanks are due to Dr. M. Kolcatt for providing a simple example to explain the limitations of the method. 


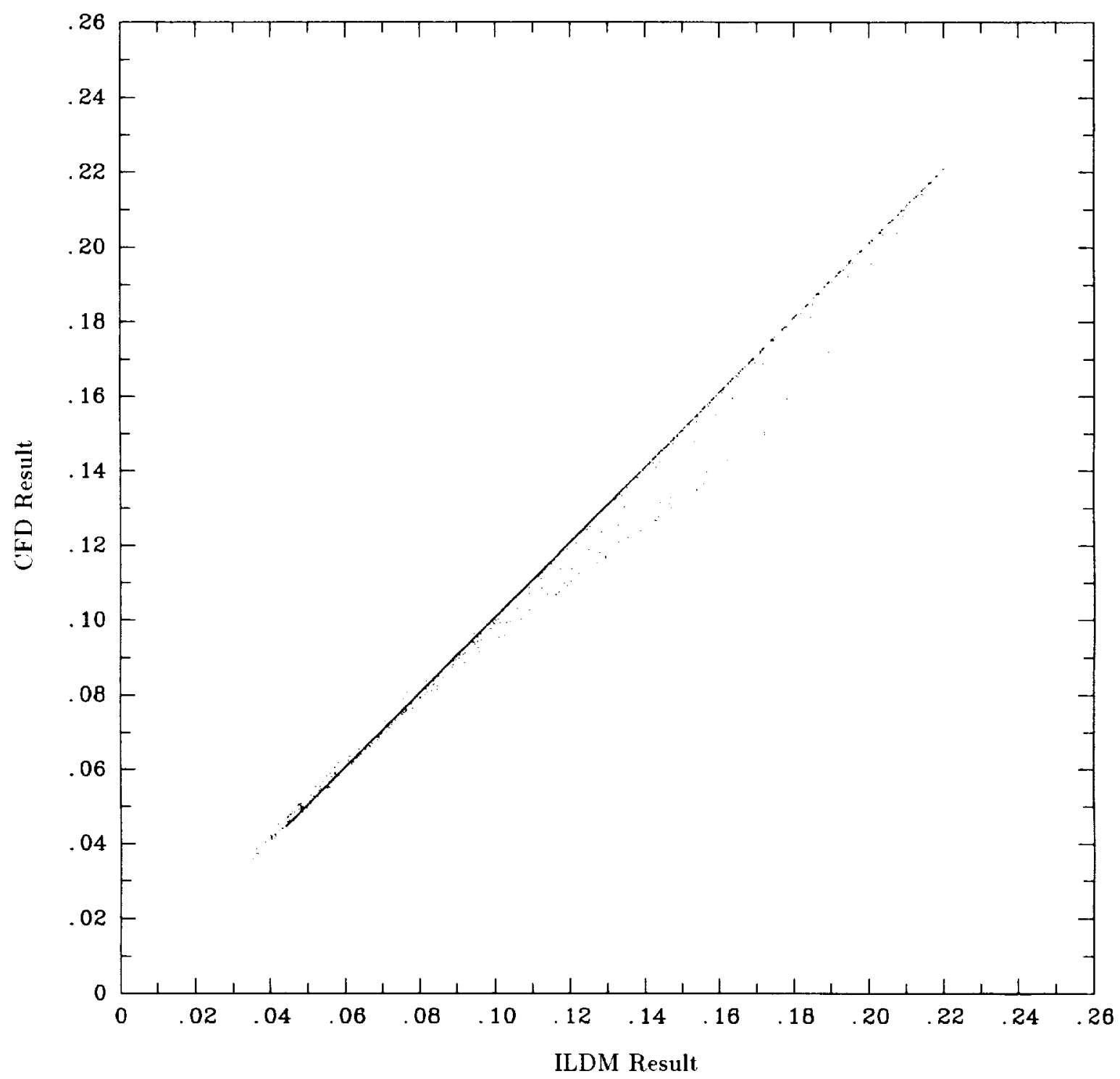

Figure 1: CFD result vs. ILDM prediction for mass fraction of $\mathrm{H} 2 \mathrm{O}$ in $\mathrm{H} 2$-air combustor. Mixture fraction range 0.005 to 0.05 . Approximately 4,200 samples. 


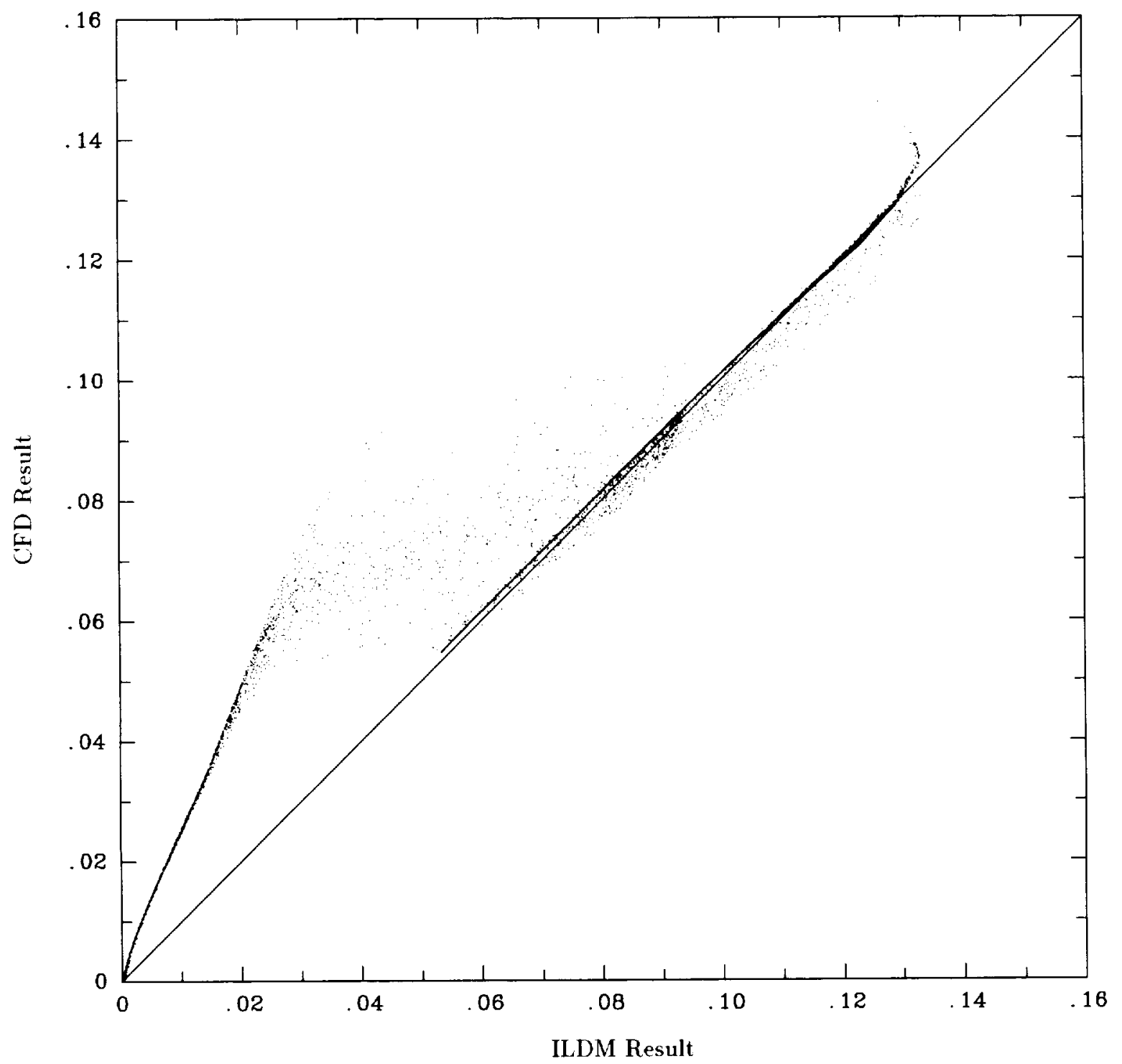

Figure 2: CFD result vs. ILDM prediction for mass fraction of $\mathrm{CO} 2$ in $\mathrm{CH} 4$-air combustor. Mixture fraction range 0.02 to 0.07 . Approximately 10,000 samples. 


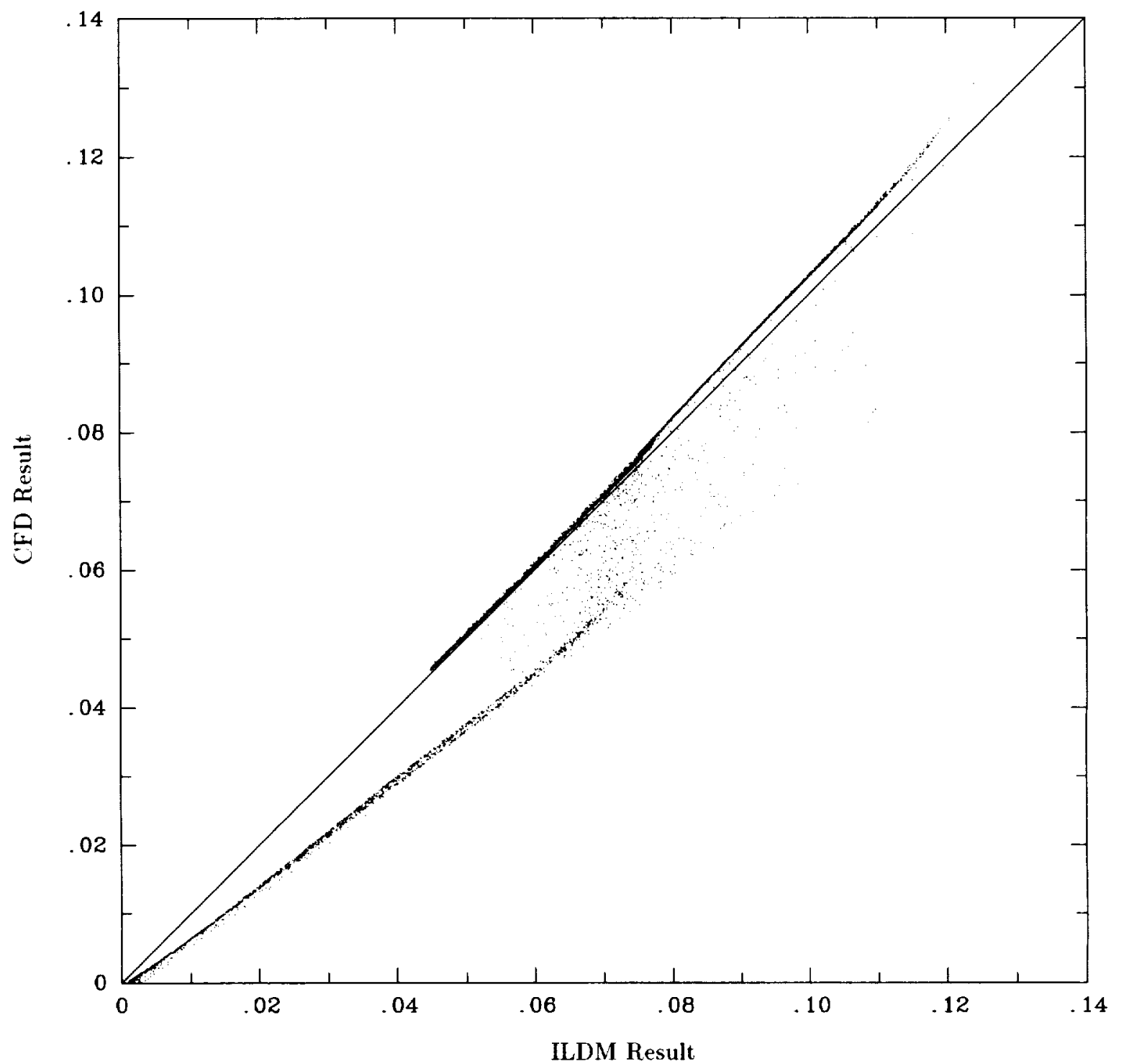

Figure 3: CFD result vs. ILDM prediction for mass fraction of $\mathrm{H} 2 \mathrm{O}$ in $\mathrm{CH} 4$-air combustor. Mixture fraction range 0.02 to 0.07 . Approximately 10,000 samples. 


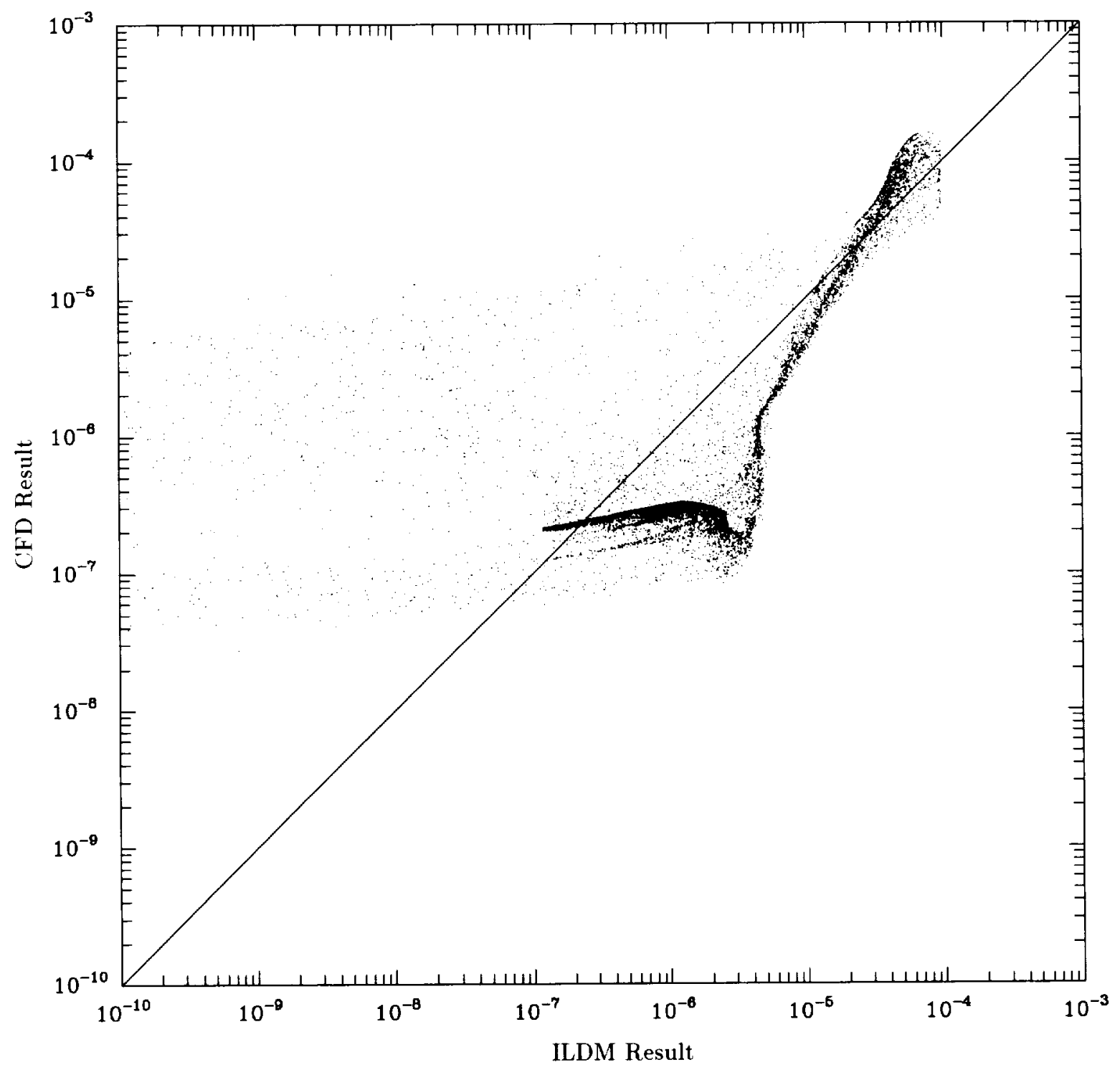

Figure 4: CFD result vs. ILDM prediction for mass fraction of NO in CH4-air combustor. Mixture fraction range 0.02 to 0.07 . Approximately 10,000 samples. 


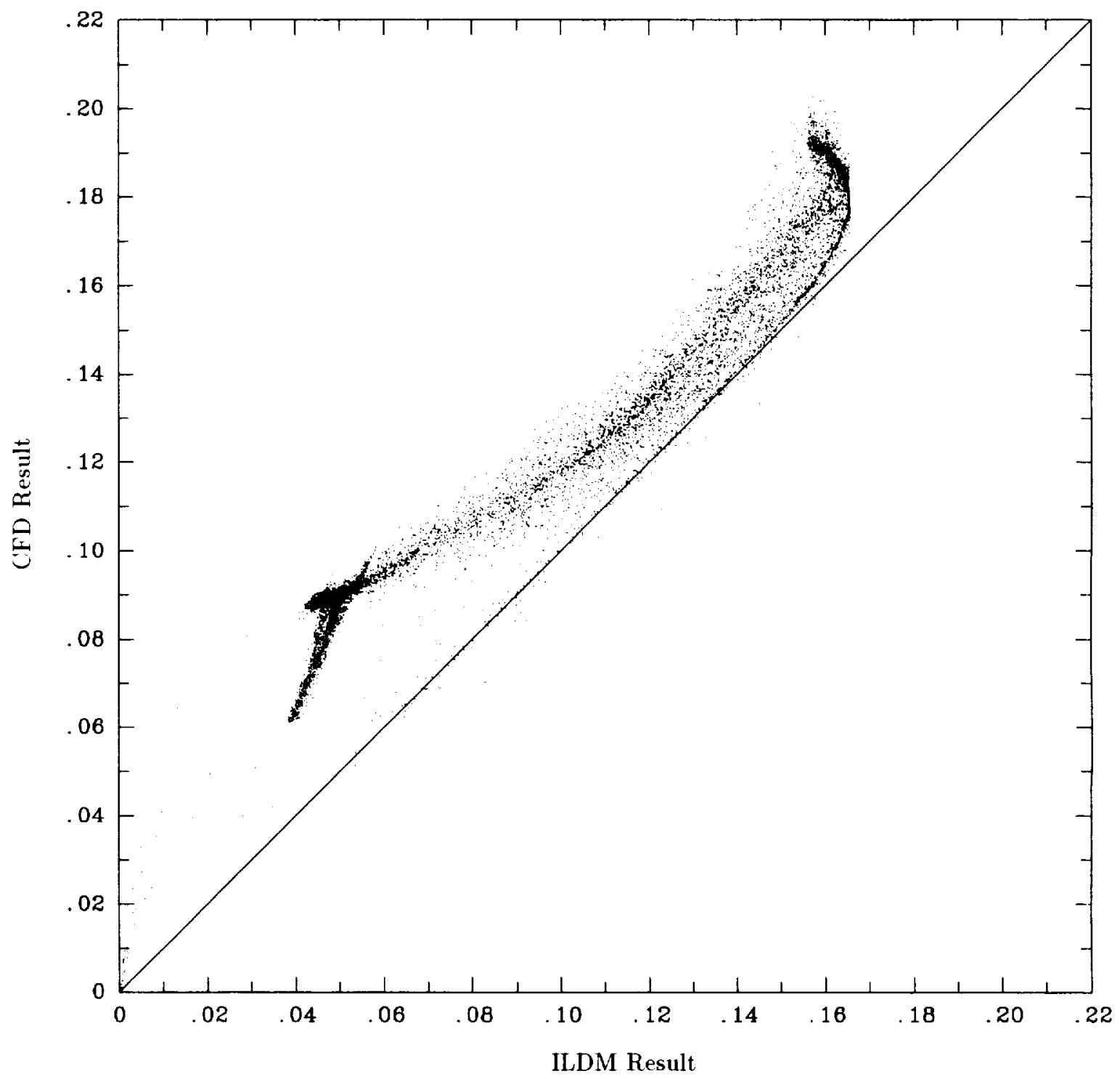

Figure 5: CFD result vs. ILDM prediction for mass fraction of $\mathrm{CO} 2$ in JetA-air combustor. Mixture fraction range 0.02 to 0.07 . Approximately 10,000 samples. 


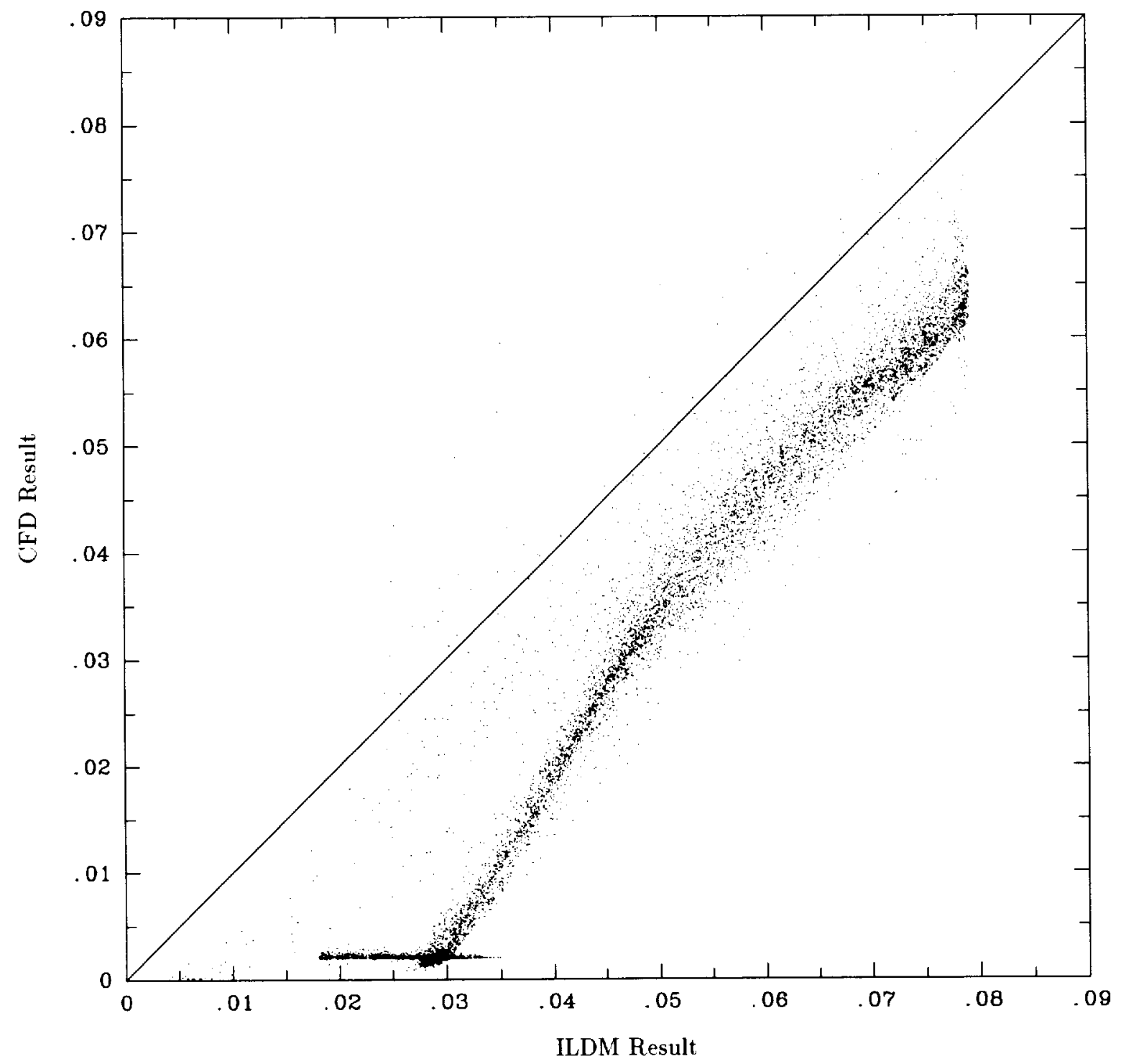

Figure 6: CFD result vs. ILDM prediction for mass fraction of $\mathrm{H} 2 \mathrm{O}$ in JetA-air combustor. Mixture fraction range 0.02 to 0.07 . Approximately 10,000 samples. 


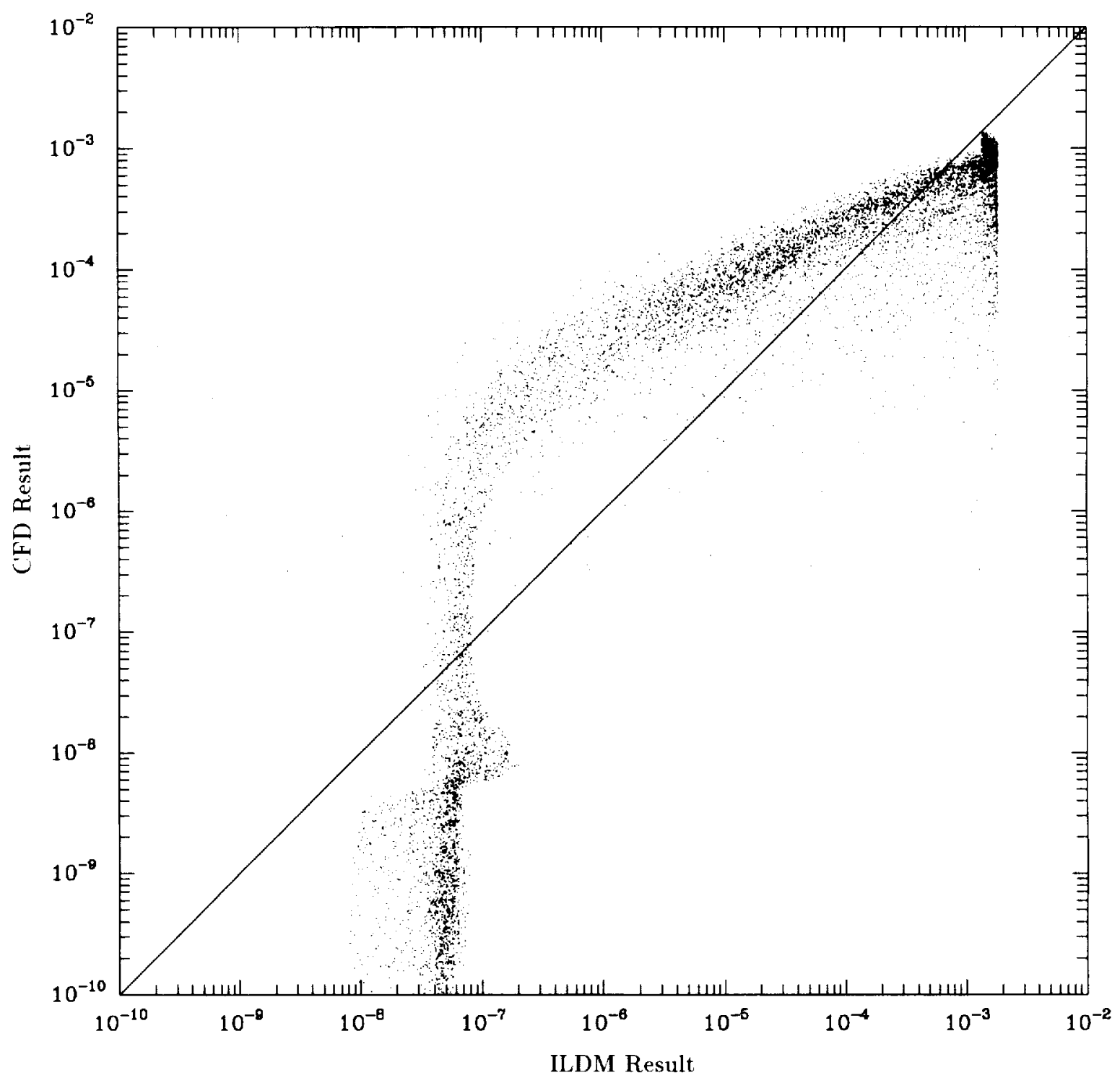

Figure 7: CFD result vs. ILDM prediction for mass fraction of NO in Jet A-air combustor. Mixture fraction range 0.02 to 0.07 . Approximately 10,000 samples. 



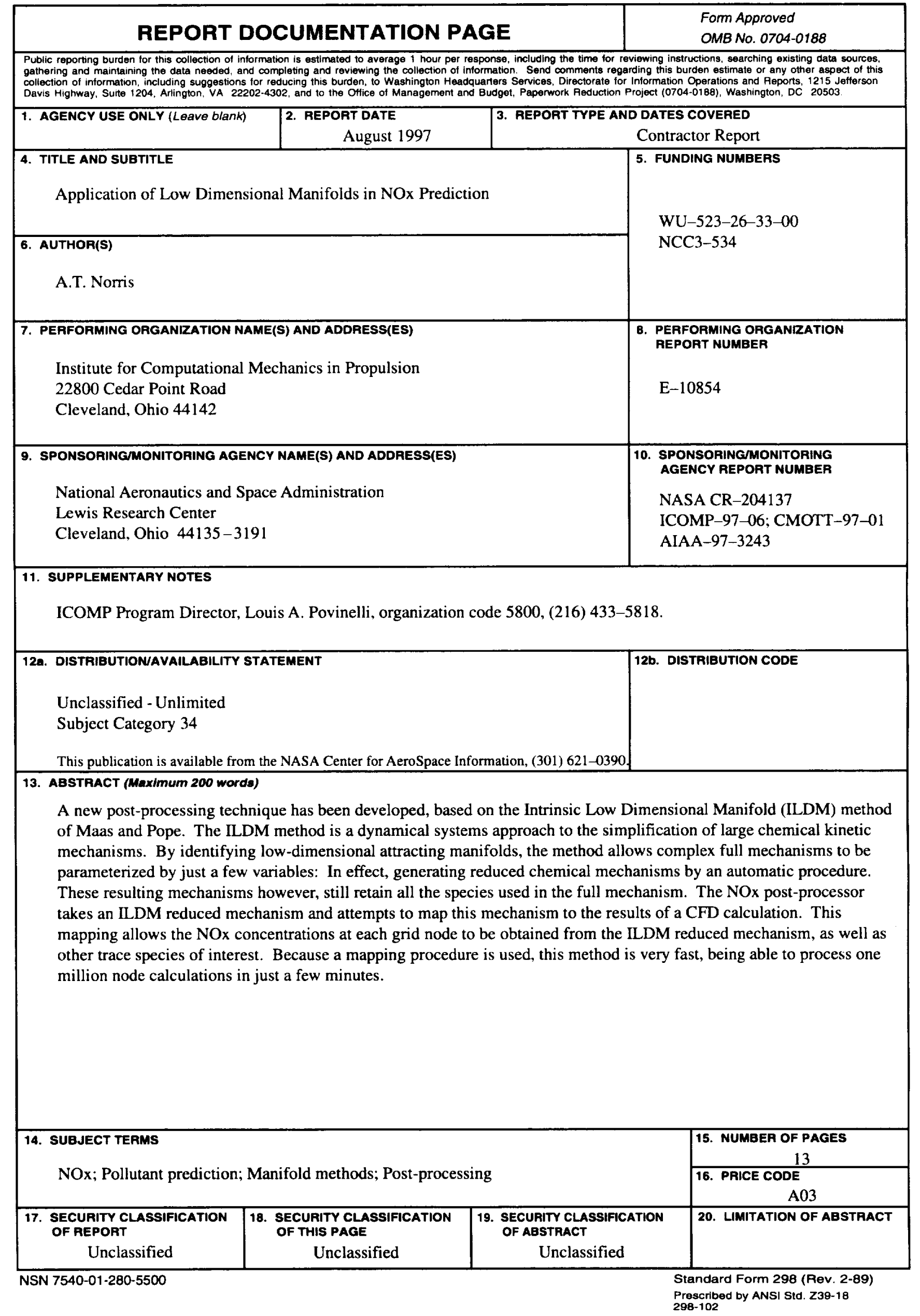

